\title{
Searching for hidden AGN in nearby star-forming galaxies with Chandra
}

\author{
P. Tzanavaris and I. Georgantopoulos
}

Institute of Astronomy \& Astrophysics, National Observatory of Athens, I. Metaxa \& V. Pavlou, Penteli 15236, Greece e-mail: pana@astro.noa.gr

Received 8 September 2006 / Accepted 17 March 2007

\section{ABSTRACT}

\begin{abstract}
Aims. We searched for X-ray signatures of AGN in a sample of star-forming, relatively early-type, nearby spiral galaxies, from the optical spectroscopic sample of Ho et al. The tight correlations between the masses of central supermassive black holes and bulge mass or velocity dispersion suggest that such galaxies are likely to host black holes in their centres. The aim is to look for X-ray signs of activity in these gas-rich environments.

Methods. We identified Chandra ACIS-S images of the X-ray counterparts of 9 sources from the optical sample. We isolated 10 individual X-ray sources closest to the optical position and calculated their X-ray luminosity, $L_{\mathrm{X}}$, and X-ray colours. Using $\mathrm{H} \alpha$ luminosities, we also calculated star formation rates. For four sources with a high number of net counts in the X-ray band, we extracted $\mathrm{X}$-ray spectra to which we fitted standard spectral models. We modelled Fe K $\alpha$ emission by Gaussians, and assessed the significance of adding such a component to a fit by means of a calibration of the standard $F$-test. For the rest of the sources, we estimated values for the intrinsic hydrogen column density, $N_{\mathrm{H}}^{\mathrm{int}}$, and the power-law photon index, $\Gamma$, which can reproduce the observed soft and hard X-ray colours.

Results. All spectral fits include a power law, a diffuse hot gas, a Galactic absorption and an intrinsic absorption component. For the nuclear sources of NGC 2782 and NGC 3310, a Fe K $\alpha$ emission-line component is included with high significance. At the same time the power-law component is flat (index $\Gamma \sim 0$ and 1.3). Both sources have high star-formation rates, with the rate for NGC 2782 being the highest in our sample.

Conclusions. The detection of Fe K $\alpha$ emission coming from a central, isolated source in NGC 2782 and NGC 3310, points towards a hidden AGN in two out of nine relatively early-type, star-forming spirals observed both with Palomar and Chandra. Larger samples need to be explored, but this evidence suggests that the presence of an AGN is likely in massive, disturbed starbursts. The contribution of X-ray emission from these AGN is small: For the central source, the bolometric luminosity is a fraction of a few $\times 10^{-5}$ of the Eddington luminosity. The X-ray flux of the central source is between 10 and $20 \%$ of the total galactic flux in the $0.2-10 \mathrm{keV}$ band.
\end{abstract}

Key words. X-rays: galaxies - galaxies: starburst - galaxies: active - galaxies: individual: NGC 2782 - galaxies: individual: NGC 3310

\section{Introduction}

The main energy source of X-ray emission from galaxies with an active galactic nucleus (AGN) is gravitational potential energy, released as gas accretes onto a central super-massive black hole (SMBH, Rees 1986; Lynden-Bell 1969; Salpeter 1964). This process leaves an imprint on X-ray spectra in the form of a power-law, perhaps due to inverse Compton up-scattering in a hot corona (Reynolds \& Nowak 2003, and references therein), although the process is not fully understood.

A number of studies suggest that all galaxies with bulges should harbour central black holes with masses that scale with bulge mass, as inferred from bulge luminosity or stellar velocity dispersion (e.g., Magorrian et al. 1998; Gebhardt et al. 2000). However, only a small fraction of bulge galaxies actually show evidence for AGN activity. For instance, in about half of the high signal-to-noise ratio optical spectra taken by Ho et al. (1997, henceforth HFS), which include several early-type spirals, there is no indication of AGN activity. The straightforward conclusion that all central black holes are dormant is puzzling, given that these are very gas-rich environments. It is possible that, although low-level AGN activity is actually taking place in these systems, its optical spectral signatures are drowned by those of circum-nuclear star formation. X-ray observations have proved to be an excellent tool for detecting the presence of a hidden AGN. In particular, nuclear Fe line emission in AGN is a unique spectral feature produced via X-ray fluorescence by matter surrounding a central black hole. Fe $\mathrm{K}$ emission has indeed been detected in several LLAGN and low-ionisation emission line regions (LINERs, Terashima et al. 2002, 2000), clinching the evidence for a central engine.

In the last few years, using Chandra, there have been a number of studies of nuclear sources in early-type galaxies. In the largest such study, Pellegrini (2005) examined the presence of nuclear sources in a sample of 50 early-type (E-Sa) nearby galaxies (most of which were from the HFS sample) and detected nuclear sources in 39 of them, with X-ray luminosities $L_{\mathrm{X}}>$ few $\times 10^{38} \mathrm{erg} \mathrm{s}^{-1}$. On the other hand, there are very few star-forming systems, such as NGC 253 (Weaver et al. 2002) and NGC 3690 (Ballo et al. 2004), which may be hosting a nuclear source suggestive of AGN emission. Similarly, most starburst interacting galaxies, such as Arp 220 and the Antennæ, also turned out to be dominated by stellar X-ray emission, without any evidence for an AGN (Fabbiano et al. 2001). Thus, until recently, few galaxies, optically classified as star-forming, had been found to harbour low luminosity AGN (LLAGN), revealed at X-ray wavelengths. However, most systems observed so far 
either do not have a prominent bulge (e.g. M 82) or may be in the process of building one, e.g. via mergers. It is then plausible that the central black hole may not be massive enough to emit copiously at X-ray energies. This could then provide an explanation why, overall, there exist no results on relatively earlytype, star-forming galaxies. For example, out of 50 galaxies in the Pellegrini (2005) sample only one, NGC 660, is classified as star-forming.

The physical and/or observational basis underlying the difficulty in identifying two clearly distinct classes of galaxies is still being intensively explored. Examples of mixed AGN/nonAGN sub-classes from the literature, which may well be related to each other, include so-called X-ray bright optically inactive galaxies (XBONGs) and "composite" galaxies. XBONGs (Georgantopoulos \& Georgakakis 2005) have optical spectra consistent with "passive" galaxies, showing no sign of AGN activity. However, their high $L_{X}$ values are hard to explain without invoking an AGN (Barger et al. 2001; Hornschemeier et al. 2001). Similarly, "composite" galaxies have high $L_{X}$ but optical spectra leading to borderline classifications between starforming and Seyfert types (Panessa et al. 2005; Moran et al. 1996). This is partly similar to cases of known AGN hosts with a significant starburst contribution to the overall energy budget. González Delgado et al. (2001) find that this is significant in a sample of 20 bright nearby type 2 Seyferts. Extending this work, these authors suggest that nuclear starbursts may well be a general part of the Seyfert phenomenon. Such starbursts may obscure low-luminosity AGN (LLAGN, $L_{\mathrm{X}} \lesssim 10^{42} \mathrm{erg} \mathrm{s}^{-1}$ ), causing a hardening of observed X-ray spectra (Fabian et al. 1998).

In the present paper we are using X-ray diagnostics to detect SMBHs hiding in relatively early-type galaxies, optically classified as star-forming. These are very gas-rich systems, with abundant fuel supply to feed a central SMBH, the presence of which has not been detected to-date. Furthermore, estimating the level of contamination of galactic X-ray flux by a central AGN is vital for understanding galaxy evolution and determining the galaxy $\mathrm{X}$-ray luminosity function.

Using the superb resolution of Chandra we are able to isolate individual, central X-ray sources for which we extract X-ray spectra. We then fit AGN models to these spectra and look for Fe K emission.

\section{The HFS optical spectroscopic sample}

HFS have carried out an extensive optical spectroscopic survey of the nuclear regions of nearby galaxies. Their main goal was to look for, and investigate, low luminosity AGN. Using the Palomar Observatory's 5-m Hale telescope, they obtained high-quality, long-slit, optical spectra of $4 \AA$ (2.5 $\AA)$ resolution in the spectral region $\sim 4230-5110 \AA(\sim 6210-6860 \AA)$ for 486 galaxies in the northern sky at a chosen magnitude limit $B_{T}=12.5 \mathrm{mag}$. By performing stellar template subtraction, they corrected the spectra for contamination by absorption lines from the stellar component. Using the emission-line intensity ratios [O III] $\lambda 5007 / \mathrm{H} \beta,[\mathrm{O}$ I] $\lambda 6300 / \mathrm{H} \alpha,[\mathrm{N} \mathrm{II}] \lambda 6583 / \mathrm{H} \alpha$ and [S II] $\lambda \lambda 6716,6731 / \mathrm{H} \alpha$, they identified $206 \mathrm{H}$ II nuclei, 52 Seyfert nuclei, 94 LINERs and 65 transition objects.

We identified all galaxies in the HFS sample which are classified both as

1. star-forming (H II nuclei), and

2. morphologically relatively early, ranging in Hubble type between $\mathrm{Sa}$ and Sc, suggesting a massive bulge and, correspondingly, a supermassive black hole.
Table 1. Chandra observation log. Columns give (1) target name, (2) Chandra sequence number, (3) observation ID, (4) exposure time, (5) Galactic neutral hydrogen column density. All observations are with ACIS-S.

\begin{tabular}{rccrr}
\hline \hline Name & Sequence no. & Obs. ID & $\begin{array}{r}t_{\text {exp }} \\
(\mathrm{ks})\end{array}$ & $\begin{array}{r}N_{\mathrm{H}}^{\text {gal }} \\
\left(10^{20} \mathrm{~cm}^{-2}\right)\end{array}$ \\
\hline NGC 278 & 600189 & 2055 & 38.3 & 13.3 \\
NGC 278 & 600190 & 2056 & 37.3 & \\
\hline NGC 891 & 600097 & 794 & 50.9 & 7.64 \\
\hline NGC 2146 & 700570 & 3131 & 9.3 & 7.30 \\
NGC 2146 & 700571 & 3132 & 9.5 & \\
NGC 2146 & 700572 & 3133 & 9.8 & \\
NGC 2146 & 700573 & 3134 & 9.6 & \\
NGC 2146 & 700574 & 3135 & 10.0 & \\
NGC 2146 & 700575 & 3136 & 9.8 & 1.76 \\
\hline NGC 2782 & 700453 & 3014 & 29.6 & 1.13 \\
NGC 3310 & 600276 & 2939 & 47.2 & 1.77 \\
NGC 4102 & 700693 & 4014 & 4.9 & 1.23 \\
NGC 4217 & 600393 & 4738 & 72.7 & 1.44 \\
NGC 4303 & 700339 & 2149 & 28.0 & \\
NGC 5905 & 700445 & 3006 & 9.6 & \\
\hline
\end{tabular}

\section{X-ray data}

We searched the Chandra archive for X-ray fields observed with the Advanced CCD Imaging Spectrometer, ACIS-S or ACIS-I, covering the optical positions of these galaxies. We identified nine sources, whose positions fall within ACIS-S fields. Details of the X-ray observations are given in Table 1.

We used type 2 event files (including only grades $0,2,3,4$, and 6) provided by the standard pipeline processing, after discarding periods of high background. Further, we used CIAO, version 3.2, to carry out astrometric corrections on these data, following the standard procedure for Chandra data ${ }^{1}$.

\section{Looking for AGN signatures}

In the X-ray band, telltale signs of AGN activity in galaxies include, generally speaking, bright nuclear sources with high $\mathrm{X}$-ray luminosities, $\log L_{\mathrm{X}} \gtrsim 42\left(\mathrm{erg} \mathrm{s}^{-1}\right)^{2}$, as well as the presence of the fluorescent $6.4 \mathrm{keV} \mathrm{Fe} \mathrm{K} \alpha$ line. Significant neutral hydrogen column densities, $\log N_{\mathrm{H}} \gtrsim 22\left(\mathrm{~cm}^{-2}\right)$, are indicative of strong intrinsic absorption, often associated with some AGN. Our aim was to identify reliable optical and X-ray counterparts for the nine sources mentioned and then search for hidden AGN by applying these criteria to X-ray counterparts. In cases were X-ray spectra could be extracted, we fitted spectral models to look for Fe $\mathrm{K} \alpha$ emission and obtain $N_{\mathrm{H}}$ estimates (Table 3). For the rest of the cases, we used the software PIMMS (v3.8a, Mukai 1993) to see which $N_{\mathrm{H}}-\Gamma$ combination could reproduce the observed X-ray colours, given the count rate, redshift and galactic column density for each source.

\subsection{X-ray and optical counterparts}

Cotton et al. (1999) have determined accurate nuclear positions for all confirmed galaxies in the Uppsala General Catalogue (UGC). For each object, they present a conservative positional uncertainty which reflects astrometric errors, as well as errors due to galaxy size and morphology. Positions from this work

\footnotetext{
${ }^{1}$ http://cxc.harvard.edu/ciao/threads/arcsec_correct ion/index.html

2 This is obviously not the case for LLAGN.
} 

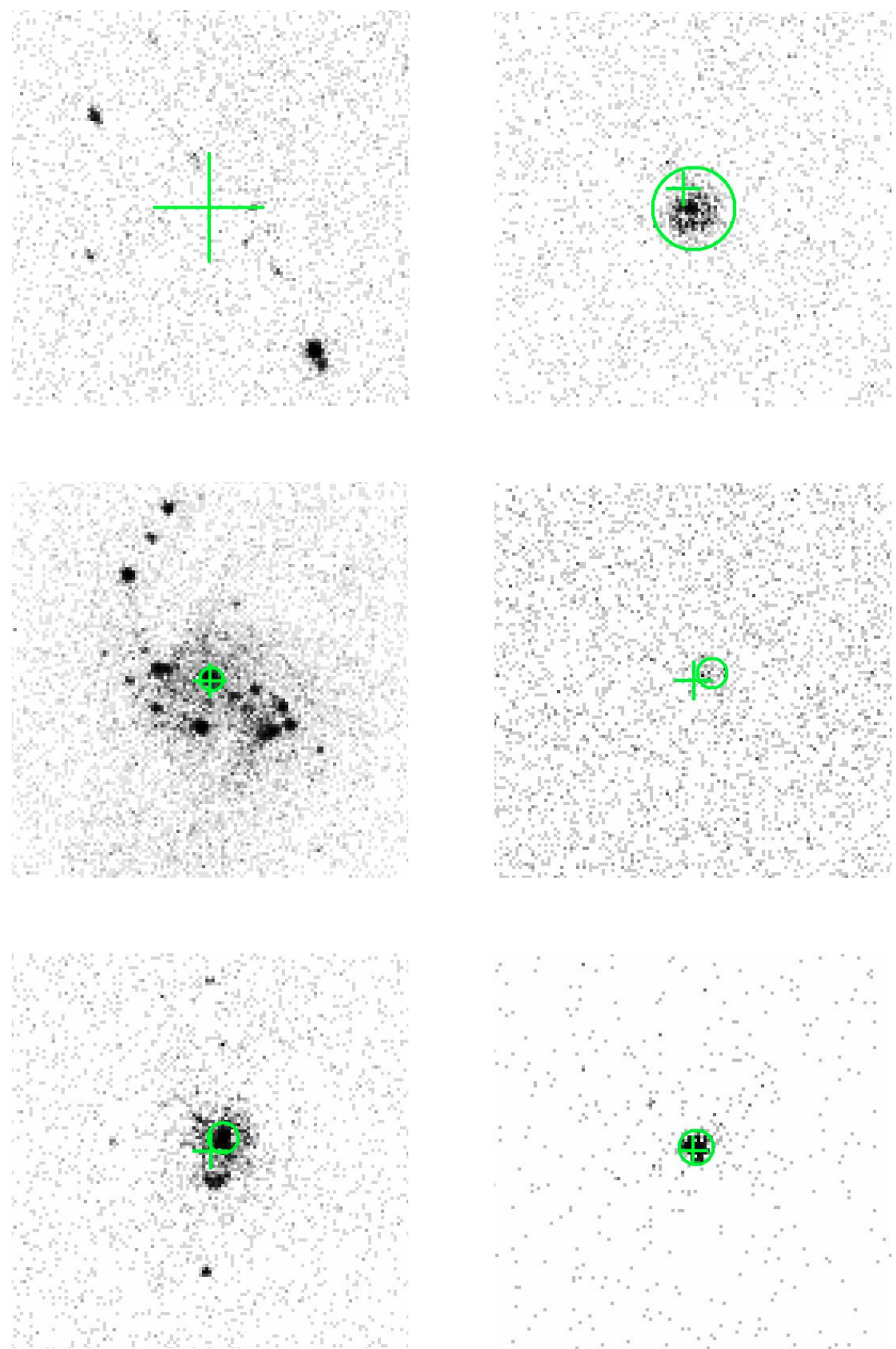

Fig. 1. Chandra/ACIS-S images of galaxies in this project. North is up and east is to the left. From left to right and top to bottom the galaxies shown are NGC 891, NGC 4303, NGC 3310, NGC 5905, NGC 2782, NGC 4102. The cross and its size represent the best optical position and its uncertainty from Cotton et al. (1999). The circular region nearest to the cross represents the best candidate for an X-ray counterpart. All images are unbinned and have a size of $61^{\prime \prime} \times 61^{\prime \prime}$.

(and 1- $\sigma$ uncertainties) are shown by the cross (and the length of its arms) in Fig. 1. As an AGN is, by definition, a nuclear object, we looked for good correspondence between these reference positions and individual X-ray sources. With the exception of NGC 891, we clearly identified X-ray counterparts for all sources, shown by the circular regions in Fig. 1. Background regions, with sufficient numbers of counts, and sources masked out, were also selected. For sources surrounded by diffuse emission, this was included in the background. In two cases, there was no clear best (closest) candidate X-ray counterpart and we chose two sources, marked by $\mathrm{U}_{\text {and }} \mathrm{L}^{3}$ in Table 2 . Details of the $\mathrm{X}$-ray/optical sample, including values for calculated quantities, are given in Table 2.

We calculated $L_{\mathrm{X}}$ by using the distances given by Ho et al. (1997) and flux values $f_{\mathrm{X}}(0.2-10.0 \mathrm{keV})$ estimated by using the tool PIMMS, (v3.8a, Mukai 1993). For the latter task we used the net count rate, a power law spectrum with index $\Gamma=1.8$

3 The "upper source", $\mathrm{U}$, is at higher declination than the "lower source", L. 

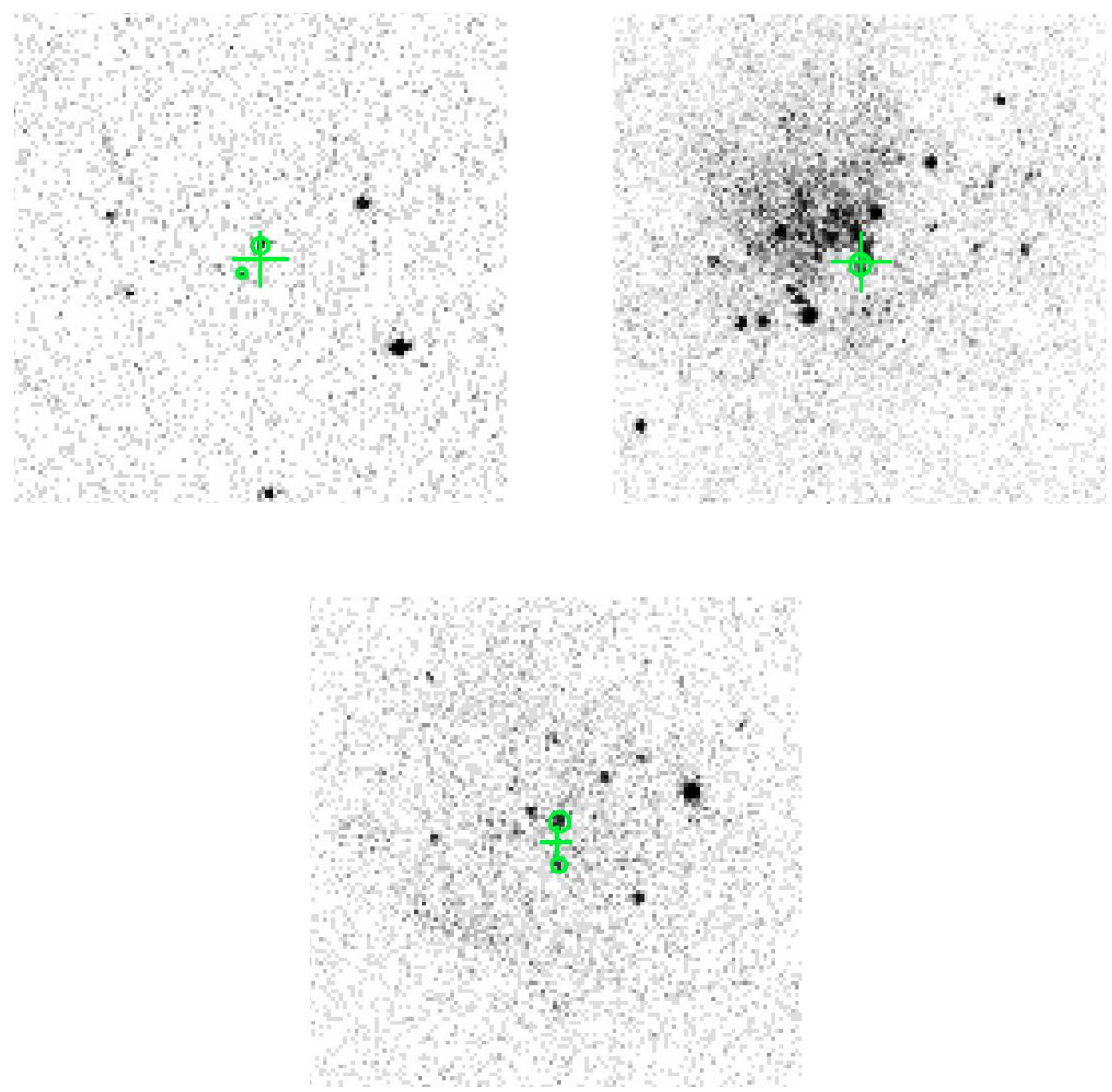

Fig. 1. continued. Images of NGC 4217, NGC 2146, NGC 278.

Table 2. HFS and Chandra galaxies. These are relatively early-type galaxies classified as H II nuclei by HFS, which have also been observed with Chandra. Shown from left to right are (1) sample identification number, (2) name, (3) right ascension and (4) declination for the X-ray source, (5) offset between X-ray and optical (Cotton et al. 1999) position, (6) B magnitude from HFS, (7) X-ray flux, (8) redshift, (9) logarithmic X-ray luminosity, (10) soft X-ray colour, (11) hard X-ray colour, and (12) circum-nuclear star formation rates calculated using H $\alpha$ luminosities taken from HFS and the relation of Kennicutt (1998).

\begin{tabular}{rcccccrcrrrr}
\hline \hline ID & Name & $\begin{array}{c}\alpha_{\mathrm{X}} \\
(\mathrm{J} 2000)\end{array}$ & $\begin{array}{c}\delta_{\mathrm{X}} \\
(\mathrm{J} 2000)\end{array}$ & $\begin{array}{c}\delta_{\mathrm{XO}} \\
\left({ }^{\prime}\right)\end{array}$ & $B$ & $\begin{array}{r}f_{\mathrm{X}} / 10^{-14} \\
(\mathrm{cgs})\end{array}$ & $\begin{array}{r}\log L_{\mathrm{X}} \\
(\mathrm{cgs})\end{array}$ & $\begin{array}{r}\mathrm{H} 1 \\
\mathrm{H} 2\end{array}$ & $\begin{array}{r}\text { SFR(H } \alpha) \\
M_{\odot} \mathrm{yr}^{-1}\end{array}$ \\
\hline 1 & NGC 278U & 005204.4 & +473304 & 2.62 & 11.5 & 0.909 & 0.0028 & 38.18 & -0.05 & -0.23 & $4.7 \times 10^{-3}$ \\
2 & NGC 278L & 005204.4 & +473258 & 2.70 & 11.5 & 0.437 & 0.0028 & 37.86 & 0.07 & -0.30 & \\
3 & NGC 891 & 022232.9 & +422045 & 0.49 & 10.9 & $<3.910$ & 0.0023 & $<38.63$ & - & - & $>1.6 \times 10^{-4}$ \\
4 & NGC 2146 & 061837.6 & +782120 & 0.29 & 11.2 & 0.547 & 0.0041 & 38.29 & 0.11 & 0.79 & $4.6 \times 10^{-2}$ \\
5 & NGC 2782 & 091405.1 & +400647 & 1.85 & 12.1 & 15.700 & 0.0090 & 40.42 & -0.02 & 0.05 & $4.9 \times 10^{-1}$ \\
6 & NGC 3310 & 103845.8 & +533011 & 0.37 & 11.2 & 26.400 & 0.0045 & 40.04 & 0.38 & -0.01 & $5.6 \times 10^{-2}$ \\
7 & NGC 4102 & 120623.0 & +524240 & 0.71 & 12.3 & 55.500 & 0.0041 & 40.28 & 0.06 & -0.12 & $8.3 \times 10^{-2}$ \\
8 & NGC 4217U & 121550.8 & +470532 & 1.74 & 10.9 & 0.231 & 0.0041 & 37.90 & 0.15 & 0.40 & $6.7 \times 10^{-4}$ \\
9 & NGC 4217L & 121551.0 & +470529 & 2.97 & 10.9 & $<0.192$ & 0.0041 & $<37.82$ & 0.12 & 0.79 & \\
10 & NGC 4303 & 122154.9 & +042825 & 3.37 & 10.1 & 21.100 & 0.0037 & 39.77 & -0.48 & -0.17 & $3.1 \times 10^{-2}$ \\
11 & NGC 5905 & 151523.3 & +553102 & 3.08 & 12.3 & 3.350 & 0.0107 & 39.90 & -0.21 & -0.27 & $1.4 \times 10^{-1}$ \\
\hline
\end{tabular}

and the Galactic neutral hydrogen column density $N_{\mathrm{H}}^{\mathrm{gal}}$ in the direction of the source (Dickey \& Lockman 1990). As can be seen in Table 2, the brightest source has $\log L_{\mathrm{X}}=40.42\left(\mathrm{erg} \mathrm{s}^{-1}\right)$, so if there is a hidden AGN, it is weak.

X-ray colours can help discriminate source types, especially when no spectra are available. We calculated the net counts in three bands: soft $(S \equiv 0.3-1 \mathrm{keV})$, medium $(M \equiv 1-2 \mathrm{keV})$, and hard $(H \equiv 2-8 \mathrm{keV})$. Following Prestwich et al. (2003), we define $\mathrm{H} 1 \equiv(M-S) / T$ and $\mathrm{H} 2 \equiv(H-M) / T$ to be the soft and hard colours, respectively. $T$ represents the total counts in all three bands combined. The X-ray colours for each source appear in Table 2. For sources with no spectra, the calculated colours are shown in the colour-colour plot of Fig. 7. Regions corresponding to different source types, taken from Prestwich et al. (2003), are marked, as explained in the figure caption.

Finally, we calculated circum-nuclear star formation rates (SFRs), using the $\mathrm{H} \alpha$ luminosities given in HFS and the relation of Kennicutt (1998). Results are presented in Table 2. The 
Table 3. Parameters from spectral fits. Shown from left to right are (1) name, (2) XSPEC model, (3) Galactic column density, (4) intrinsic column density from XSPEC, (5) photon index for power law component, (6) peak energy of gaussian emission line, (7) equivalent width of the line, (8) plasma temperature, (9) $\chi^{2} /$ degrees of freedom, (10) unabsorbed $L_{\mathrm{X}}$ from power law and (11) from thermal component (both in units $\left(10^{40} \mathrm{erg} \mathrm{s}^{-1}\right)$. The spectral models are: $\mathrm{PL}=$ power law continuum; $\mathrm{M}=$ MEKAL thermal plasma (solar abundances); RS = Raymond-Smith thermal plasma (solar abundances); $\mathrm{G}=$ Gaussian emission line; $\mathrm{W}=$ photoelectric absorption (Wisconsin cross-sections); $\mathrm{ZW}=$ redshifted $\mathrm{W}$. Uncertainties give $90 \%$ confidence intervals.

\begin{tabular}{llccccccccc}
\hline \hline Name & Model & $\begin{array}{c}N_{\mathrm{H}}^{\mathrm{gal}} \\
\left(10^{20} \mathrm{~cm}^{-2}\right)\end{array}$ & $\begin{array}{c}N_{\mathrm{H}}^{\text {int }} \\
\left(10^{22} \mathrm{~cm}^{-2}\right)\end{array}$ & $\Gamma$ & Fe K $\alpha$ & $\begin{array}{c}E W \\
(\mathrm{keV})\end{array}$ & $\begin{array}{c}k T \\
(0.1 \mathrm{keV})\end{array}$ & $\chi^{2} /$ d.o.f. & $L_{\mathrm{X}}(\mathrm{PL})$ & $L_{\mathrm{X}}(\mathrm{th})$ \\
\hline NGC 2782 & (PL+M+G)*ZW*W & 1.76 & $<0.07$ & $0.0_{-0.3}^{+0.6}$ & 6.4 & $1.5_{-0.7}^{+0.7}$ & $9.62_{-0.07}^{+0.19}$ & $32.3 / 31$ & $4.81_{-2.09}^{+3.17}$ & $0.59_{-0.15}^{+0.08}$ \\
NGC 3310 & (PL+M+G)*ZW*W & 1.13 & $0.23_{-0.05}^{+0.05}$ & $1.3_{-0.3}^{+0.2}$ & 6.4 & $0.3_{-0.2}^{+0.2}$ & $\ldots$. & $68.6 / 83$ & $1.95_{-0.93}^{+1.69}$ & $0.42_{-0.35}^{+0.70}$ \\
NGC 4102 & (PL+RS+G)*ZW*W & 1.77 & $0.19_{-0.65}^{+0.33}$ & $1.7_{-1.3}^{+0.9}$ & $\ldots$ & $\ldots$ & $0.7_{-0.4}^{+0.2}$ & $18.6 / 11$ & $1.52_{-1.25}^{+1.08}$ & $2.99_{-2.78}^{+11.94}$ \\
NGC 4303 & (PL+M)*ZW*W & 1.67 & $<0.03$ & $1.9_{-0.2}^{+0.3}$ & $\ldots$ & $\ldots$ & $5.37_{-0.06}^{+0.09}$ & $35.0 / 31$ & $0.17_{-0.03}^{+0.06}$ & $0.14_{-0.02}^{+0.03}$ \\
\hline
\end{tabular}

purpose of this was to investigate whether there is any link between high SFRs and AGN activity, as the gas fuelling star formation may also sustain an AGN.

\subsection{X-ray spectra}

For all individual X-ray sources identified and having $>60$ net counts we extracted spectra by using the CIAO 3.2 script psextract. Four spectra were extracted in all, the faintest extracted source having 333 counts. The spectra were binned to have 15 counts per bin and analysed with the XSPEC software package, version 12.2.0. Details of the spectral results are given in Table 3. All spectra were fitted with models which included at least a power law and Galactic absorption. There is a significant range in spectral shapes (see below). Any intrinsic absorption appears to be no more than a few $\times 10^{21} \mathrm{~cm}^{-2}$. At low energies a thermal component is needed to account for a pronounced spectral "bump".

In two spectra there is clear indication of line emission between 6 and $7 \mathrm{keV}$. It is common practice to estimate the significance of including an emission line as an additive component to the spectral model by means of a standard $F$-test. However, this can be misleading. As explained by Protassov et al. (2002), one of the conditions for the use of $F$-statistics stipulates that the null values of additional parameters introduced may not be on the boundary of the set of possible parameter values. As the line flux must be non-negative, its null value is zero, which is the boundary of non-negative numbers. In such cases, one has to modify the standard procedure by performing a calibration of the $F$ test applied on the observed spectrum ( $F$-test calibration, FC). Briefly, one carries out Monte Carlo simulations of spectra using the parameters for the best null model fit, i.e. without an emission line, and the same instrument response, background, exposure time and binning as for the observed spectrum. Each simulated spectrum is fitted twice, once with a model that includes the emission line, and once with a model without the emission line. The $F$-test is applied to each fitting pair, and the ensemble of such $F$-test results is compared to the single $F$-statistic value and associated probability result from the pair of fits to the observed spectrum, thus providing an estimate of the reliability of the calculated significance of the observed $F$-statistic. In our case, we applied this method by carrying out 2000 Monte Carlo simulations for each observed spectrum.

We now discuss each case in greater detail.

\subsubsection{NGC 891}

Optical imaging shows that this source has an edge-on orientation. This may be one reason why, as can be seen in Fig. 1, there

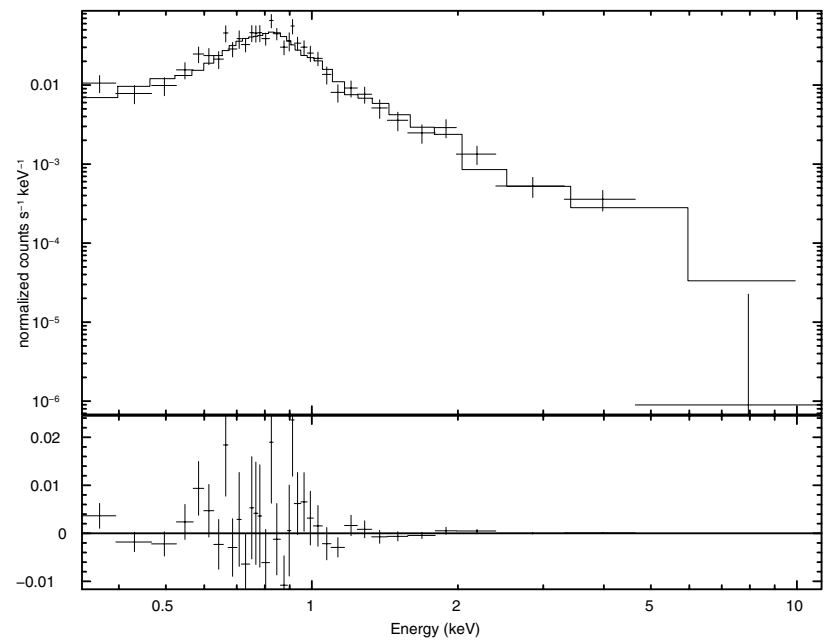

Fig. 2. Fitted Chandra-ACIS X-ray spectrum of NGC 4303.

is no candidate X-ray counterpart in the ACIS field corresponding to this source. Accordingly, the flux and luminosity values in Table 2 are upper limits, and we do not discuss this source any further.

\subsubsection{NGC 4303}

The isolated X-ray source shows 728 net counts. The centre of the selected circular source region is at $\gtrsim 3^{\prime \prime}$ from the optical position. Binning of the X-ray image reveals that the X-ray/optical pair appears roughly in the centre of a ring of diffuse X-ray emission. HFS classify this galaxy as an $\mathrm{H}$ II nucleus, although their values for $[\mathrm{N} \mathrm{II}] / \mathrm{H} \alpha$ and $[\mathrm{S} \mathrm{II}] / \mathrm{H} \alpha$ favour an AGN classification. This is because HFS give less weight to the $[\mathrm{N} \mathrm{II}] / \mathrm{H} \alpha$ ratio due to the possibility of selective nitrogen enhancement in galactic nuclei, and the $[\mathrm{S} \mathrm{II}] / \mathrm{H} \alpha$ value is borderline between $\mathrm{H}$ II and AGN.

The X-ray spectrum (Fig. 2) is best fitted with a redshifted power-law plus hot diffuse gas model (MEKAL), with only the default parameters kept fixed. There is no indication of emission in the spectral region around $6.4 \mathrm{keV}$.

\subsubsection{NGC 3310}

More than ten discrete X-ray sources, embedded in diffuse emission, can be seen in the ACIS image of this galaxy. One of the sources corresponds extremely well with the optical centre, and has been isolated for further study here. As this isolated source shows 1538 net counts, a high-quality spectrum can be extracted. 


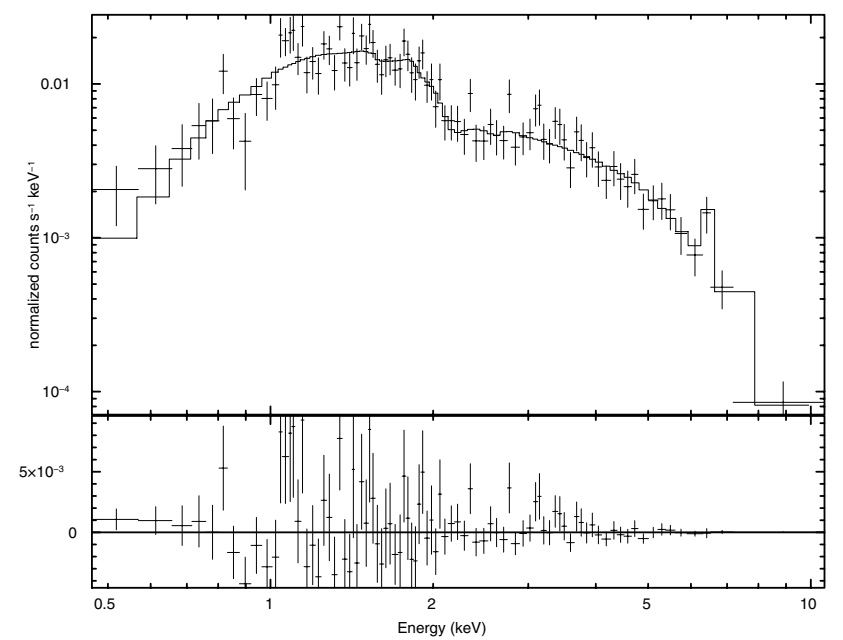

Fig. 3. Fitted Chandra-ACIS X-ray spectrum of NGC 3310.

Up to $\sim 7 \mathrm{keV}$, the continuum is well defined, making a prominent emission feature clearly noticeable at $6.4 \mathrm{keV}$ at a level of $\sim 2 \sigma$ above the local continuum. The spectrum (Fig. 3 ) is well fitted with a power law, a thermal component and moderate intrinsic absorption. The power law is flatter than the canonical average for AGN. Fixing $\Gamma$ at a canonical AGN value of 1.8 leads to a somewhat worse $\chi^{2}$, but the intrinsic column density remains virtually unchanged.

The best fit (reduced $\chi^{2} \equiv \chi_{v}^{2} \sim 0.8$ ) includes a gaussian component for an emission line fixed at $\sim 6.4 \mathrm{keV}$ with an equivalent width $(E W)$ of $0.3 \mathrm{keV}$. A similar, if slightly poorer, fit is produced if the line energy and Gaussian $\sigma$ of the line profile are left free. In this case, the best fit energy is $6.4 \pm 0.1 \mathrm{keV}$. This cannot be achieved with a $6.7 \mathrm{keV}$ line. Both the energy and Gaussian $\sigma$ must then be fixed, but this produces a poorer fit than without an emission line at all, and thus no $F$-test can be applied for such a line. Plotting data and fits shows clearly that only a narrow line at $6.4 \mathrm{keV}$ is able to fit the sharp feature at $>6 \mathrm{keV}$ above the well defined continuum.

Indeed, the calibrated $F$-test suggests that the fitted emission line is statistically significant: the $F$-statistic value for the observed spectrum is 4.8 , with random probability 0.03 . FC shows that more than $99 \%$ of the simulated spectra lead to a smaller $F$-value, and, correspondingly, higher random probabilities.

\subsubsection{NGC 5905}

This is the most distant source in the sample. The level of net counts (40) is low, precluding the extraction of a useful spectrum. Both X-ray colours are quite soft, suggesting a source with $\Gamma \sim 2.5$. In the colour-colour diagram of Fig. 7 this source is located in the low-mass X-ray binary (LMXB) region.

\subsubsection{NGC 2782}

The X-ray source has 597 net counts. It can be seen in Fig. 4 that the extracted spectrum has a pronounced bump at energies below $\sim 1.5 \mathrm{keV}$ which is well fitted by a hot diffuse-gas model. The high energy region is fitted by a power law which appears to be flat. The best fit $\left(\chi_{v}^{2}=1.0\right)$ requires a gaussian component at $6.4 \mathrm{keV},(E W \sim 1.5 \mathrm{keV})$ with a high-significance $F$-statistic value of 17.0 and associated random probability $3 \times 10^{-4}$. FC shows that all 2000 simulated spectra lead to smaller $F$-values.

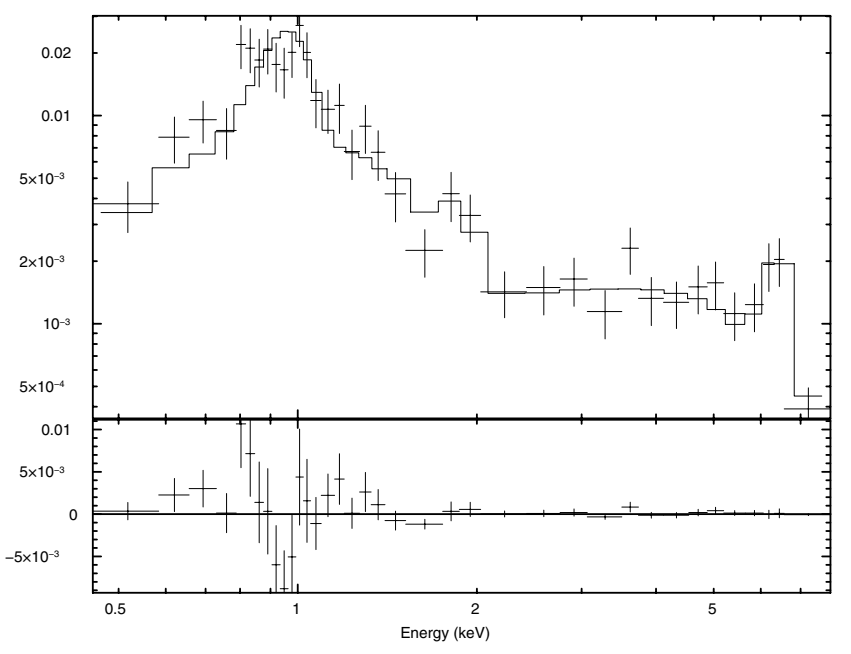

Fig. 4. Fitted Chandra-ACIS X-ray spectrum of NGC 2782.

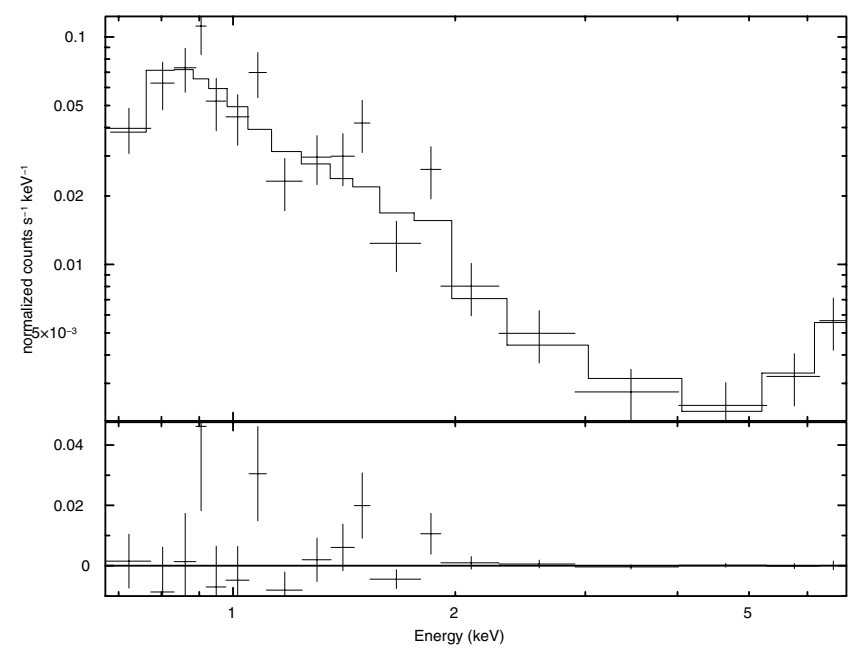

Fig. 5. Fitted Chandra-ACIS X-ray spectrum of NGC 4102.

As in the case of NGC 3310, if the line energy is left free, XSPEC places the line at $6.4 \pm 0.1 \mathrm{keV}$. Fitting a line at 6.7 , rather than $6.4, \mathrm{keV}$ requires fixing all line components, the fit is clearly poorer $\left(\chi_{v}^{2}=1.4\right)$ and, as the number of degrees of freedom is the same as without a line, the $F$-test is inapplicable.

\subsubsection{NGC 4102}

This is a clear, isolated X-ray source with sub-arcsecond separation from its optical counterpart, and 333 net counts. The extracted X-ray spectrum is shown in Fig. 5. A power-law fit provides a mediocre fit, which is somewhat improved by the addition of a Raymond-Smith hot, diffuse-gas model ( $F$-test probability 0.1 ). At energies $\gtrsim 5 \mathrm{keV}$ the continuum appears to be sloping upwards. This feature can be fitted by a Gaussian at $\sim 9 \mathrm{keV}$ with an $E W \gtrsim 450 \mathrm{keV}$. Both values are not well constrained but lead to a much improved fit. To test the reality of this feature, we used several, distinct background regions and performed a separate spectral extraction, but this feature persists. At a lower grouping of 5 counts per bin this feature looks more like a noisy broad bump around $7 \mathrm{keV}$ with an equivalent width $\sim 200 \mathrm{keV}$. However, this line cannot be shown to be statistically significant. We thus do not take this feature to be indicative of 
Table 4. $\mathrm{H} 1$ and $\mathrm{H} 2$ results. Shown are $N_{\mathrm{H}}^{\text {int }}-\Gamma$ pairs which, assuming a pure power-law spectrum, reproduce the X-ray colours calculated from the X-ray image for sources for which no spectra have been extracted. From left to right columns give (1) name, (2) Galactic column density, (3) intrinsic column density, and (4) photon index for power law component. The last column (5) indicates the type of object consistent with the X-ray colours, from a comparison with Fig. 4 of Prestwich et al. (2003) (see Fig. 7). As mentioned in the text, there are two alternatives for NGC 2146.

\begin{tabular}{lrrrr}
\hline \hline Name & $\begin{array}{r}N_{\mathrm{H}}^{\text {gal }} \\
\left(10^{20} \mathrm{~cm}^{-2}\right)\end{array}$ & $\begin{array}{r}N_{\mathrm{H}}^{\text {int }} \\
\left(10^{22} \mathrm{~cm}^{-2}\right)\end{array}$ & $\Gamma$ & Type \\
\hline NGC 278U & 13.3 & 0 & 2.7 & LMXB \\
NGC 278L & 13.3 & 0 & 2.3 & LMXB \\
NGC 2146 & 7.30 & 4.0 & 1.8 & AGN \\
& & 1.0 & 0.3 & HMXB \\
NGC 4217U & 1.23 & 1.3 & 1.0 & HMXB \\
NGC 4217L & 1.23 & 2.5 & 1.0 & HMXB \\
NGC 5905 & 1.44 & 0 & 2.5 & LMXB \\
\hline
\end{tabular}

Fe $\mathrm{K} \alpha$ or $\beta$ emission near $7 \mathrm{keV}$, although, the excellent agreement between the optical and X-ray positions would make this object a good hidden AGN candidate.

\subsubsection{NGC 4217}

There are two isolated X-ray sources, $U$ and L, closest to the optical position. These have 21 and 9 counts, respectively, which are very low for a useful spectrum to be extracted. Additionally, the flux and luminosity information for source $\mathrm{L}$ are $2 \sigma$ upper limits. Both sources fall in the the high-mass X-ray binary (HMXB) region of Fig. 7. Their X-ray colours are hard and are suggestive of a relatively flat photon index $(\Gamma \sim 1)$ and high intrinsic absorption (few $\times 10^{22} \mathrm{~cm}^{-2}$ ).

\subsubsection{NGC 2146}

As in the case of NGC 3310, the ACIS image of this galaxy shows several discrete point sources embedded in diffuse emission. The source that best corresponds to the optical position has only 32 net counts, but the positional agreement is the best among all of our X-ray/optical pairs. Given its X-ray colours, this source may have an intrinsic $\Gamma \sim 1.8$ and $N_{\mathrm{H}} \sim 4 \times$ $10^{22} \mathrm{~cm}^{-2}$, consistent with an AGN with high intrinsic absorption. Alternatively, the source is also consistent with a flat $\Gamma \sim$ $0.3, N_{\mathrm{H}} \sim 1 \times 10^{22} \mathrm{~cm}^{-2}$, and falls in the HMXB region of Fig. 7 .

\subsubsection{NGC 278}

As in the case of NGC 4217, there are two candidate X-ray counterparts, $\mathrm{U}$ and $\mathrm{L}$, roughly equidistant from the optical position, with 60 and 29 counts, respectively. The X-ray colours for both sources are quite soft, suggesting no intrinsic absorption, steep photon indices (2.3-2.7), and are consistent with LMXBs.

\section{Discussion}

\subsection{NGC 2782 and 3310 revisited}

There is only a handful of star-forming galaxies known to harbour a LLAGN, revealed at X-ray wavelengths. Out of nine HFS sources which fall within ACIS-S fields, we extracted four spectra. In two out of these, we detect a $6.4 \mathrm{keV}$ Fe $\mathrm{K} \alpha$ line with high significance. In contrast, the data for these isolated, central sources do not corroborate a $6.7 \mathrm{keV}$ emission line, often associated with star-formation (Wang et al. 2002; Strickland 2005). Due to the small number of relatively early-type H II nuclei from HFS, whose positions have been observed in the X-ray band, the significance of the detection fraction (2 out of 9) remains, of course, unclear.

The NGC 2782 detection has the highest significance although the spectrum is noisier, the continuum less well-defined, the model goodness-of-fit and the optical/X-ray positional correspondence poorer. In spite of these caveats, the isolated central $\mathrm{X}$-ray source has the highest $L_{\mathrm{X}}$ in our sample. Furthermore, the detection of a hidden AGN in this object would not come as a surprise. Within the context of ROSAT observations, the possibility of a LLAGN was already considered by Schulz et al. (1998) and the galaxy is in fact classified as a Seyfert 2 by Véron-Cetty \& Véron (2003). The galaxy is number 215 in Arp's Atlas of Peculiar Galaxies (Arp 1966) and has Hubble type classification SAB(rs)a pec (Wehner \& Gallagher 2005). Amongst nearby spirals, it harbours one of the most powerful, M 82-like, nuclear starbursts (Balzano 1983; Devereux 1989), fuelled by a massive, gas-rich nuclear bar, driving molecular gas towards the central regions at large gas-inflow rates, $>1 M_{\odot} \mathrm{yr}^{-1}$ (Jogee et al. 1999). A pair of prominent $\mathrm{H}$ I and optical tails surrounding an optical disk with three ripples (Smith 1991; Smith et al. 1999) suggest that the starburst may have been triggered by an interaction or merger (Smith 1994). The presence of large amounts of gas and an efficient fuelling mechanism indicate that there should be plenty of material both for feeding an AGN co-existing with the powerful starburst and for absorbing soft X-rays so as to produce the hard, flat power-law and a Fe K $\alpha$ line with a moderately high $E W \gtrsim 1 \mathrm{keV}$. Indeed, the $\mathrm{H} \alpha$-calculated SFR for this source is the highest among all sources in our sample (Table 2).

Levenson et al. (2002) have shown that $\mathrm{Fe} \mathrm{K} \alpha$ equivalent widths correlate with geometry: larger EWs imply an "AGNType 2" view, whilst smaller EWs an "AGN-Type 1" view, in which the X-ray continuum is viewed directly. Although, their models are for heavily obscured, Compton thick AGN, high intrinsic column densities are certainly plausible in this galaxy.

The third highest $L_{X}$ in our sample is for NGC 3310. This galaxy (Arp 217), is also an M 82-class starburst, of type SAB(r)bc pec (de Vaucouleurs et al. 1991). Its peculiar optical morphology and tidal debris (Wehner \& Gallagher 2005) suggest that the starburst has been triggered by a merger with either a gas-rich dwarf companion (Balick \& Heckman 1981; Smith et al. 1996) or a similar-size disk (Kregel \& Sancisi 2001). The nuclear spectrum has been explained by using only photoionisation by hot stars (Pastoriza et al. 1993). Until now there has been no indication of AGN activity in any wavelength band. In particular, work with ROSAT (Zezas et al. 1998) and XMM-Newton (Jenkins et al. 2004) has found no indication of X-ray emission due to an AGN in this object. However, ROSAT has lower sensitivity and resolution than Chandra, whilst Jenkins et al. (2004) have excluded the energy range $>6 \mathrm{keV}$ in the analysis of the $X M M-N e w t o n$ data. It would then be the first time that this object can be searched for Fe K $\alpha$ emission, and the evidence suggests that such emission has been detected. The $\mathrm{H} \alpha$-calculated star-formation rate for this source, is the fourth highest in our sample.

We calculated the black hole mass, $M_{\bullet}$, and the corresponding Eddington luminosity, $L_{\text {Edd }}$, in these two galaxies by using velocity dispersion, $\sigma$, values from McElroy (1995) and the $M_{\bullet}-\sigma$ relation of Ferrarese \& Ford (2005, Eq. (20)). We estimated the bolometric luminosity of the isolated, central sources, 


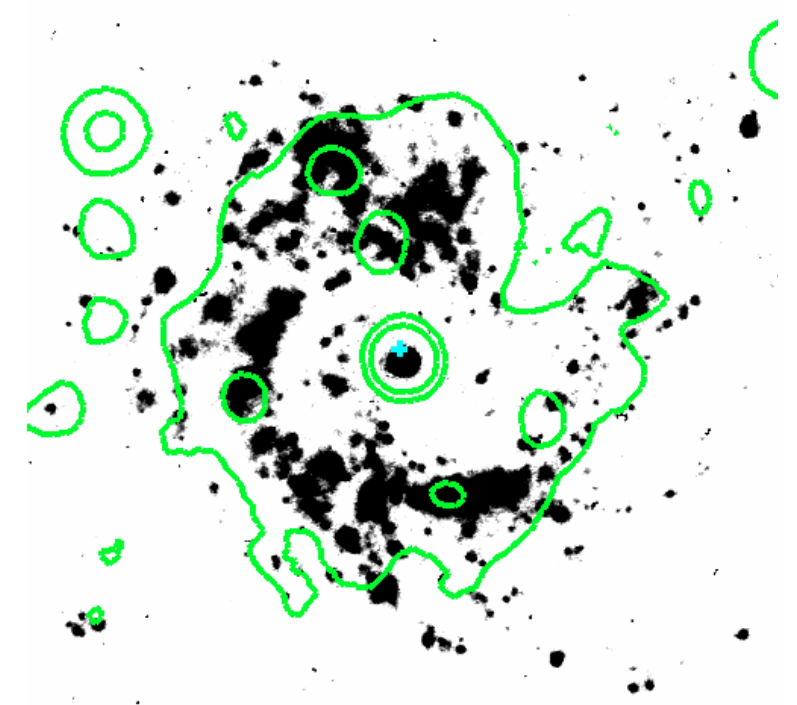

Fig. 6. X-ray contours for Chandra-ACIS X-ray image of NGC 4303 overlayed on $\mathrm{H} \alpha$ image (Knapen et al. 2004) covering $\sim 240^{\prime \prime} \times 240^{\prime \prime}$. The contours have been obtained after $4 \times 4$ binning of the X-ray image. The position of the centroid from Cotton et al. (1999) is indicated by the cross.

assuming $L_{\mathrm{bol}} \approx 10 L_{\mathrm{X}}$ (Nicastro et al. 2003). This gives $L_{\text {bol }} / L_{\text {Edd }} \sim 6 \times 10^{-5}$ and $9 \times 10^{-5}$ for NGC 2782 and NGC 3310 , respectively. Thus, the luminosity due to black hole accretion is significantly sub-Eddington. Furthermore, the fraction of X-ray flux coming from the central source, to that from the whole galaxy is $\sim 20 \%$ and $10 \%$ for NGC 2782 and NGC 3310, respectively. It follows, that any AGN contribution to the energy output of these objects is small.

\subsection{The case of NGC 4303}

We believe the case for a hidden AGN in this galaxy is strong, although it cannot be corroborated by detection of Fe emission. The galaxy has morphological type SAB(rs)bc (de Vaucouleurs et al. 1991) and is classified as Seyfert 2 by Véron-Cetty \& Véron (2003). Colina \& Wada (2000) and Colina et al. (1997) also label the galaxy as "active". It has a large-scale, outer bar (Laine et al. 2002), as well as a smaller inner bar, surrounded by a $\sim 0.5 \mathrm{kpc}$ circum-nuclear starburst ring (Colina \& Wada 2000; Pérez-Ramírez et al. 2000; Colina et al. 1997). Using ROSAT observations, Tschöke et al. (2000) have detected several point sources across the face of this spiral which they attribute to HMXBs, tracing actively star-forming H II regions. In Fig. 6 we overplot contours from the binned X-ray image on an $\mathrm{H} \alpha$ image of NGC 4303 (Knapen et al. 2004). As can be seen from this figure, as well as Fig. 1 of Jiménez-Bailón et al. (2003), the spiral pattern is traced well in the X-rays. The centermost contours in Fig. 6 most likely trace the galaxy's circum-nuclear ring which has been resolved with HST in the ultra-violet band (UV) by Colina et al. (1997) and studied extensively by Jiménez-Bailón et al. (2003). In spite of detailed evolutionary synthesis modelling, these authors cannot unambiguously distinguish among different possibilities for explaining the central X-ray emission, including a number of hidden AGN scenarios, a central star cluster, or Ultra-Luminous X-ray (ULX) sources.

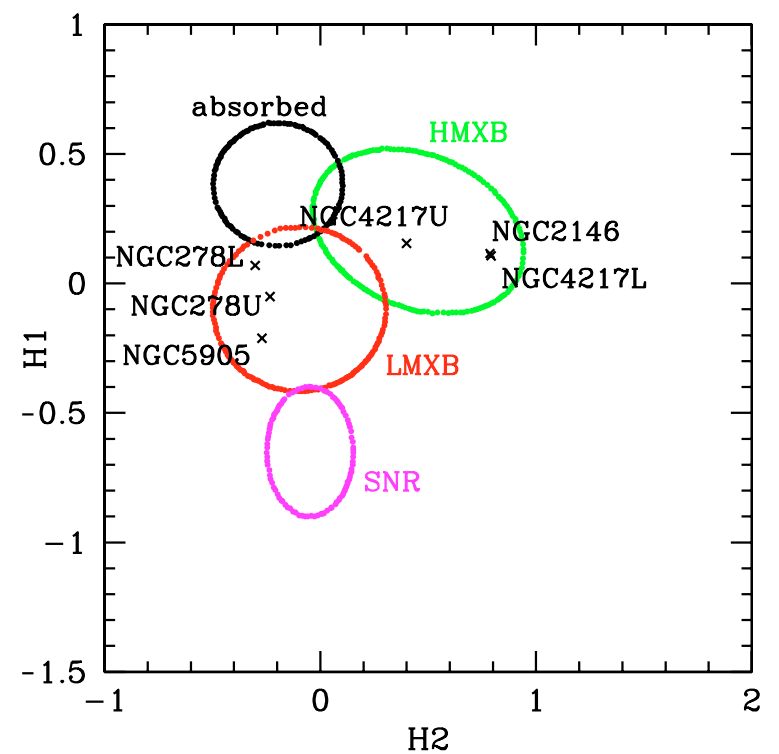

Fig. 7. X-ray colour-colour plot. The coloured contours, taken from Prestwich et al. (2003), indicate regions for LMXBs (red), HMXBs (green), absorbed sources (black), and thermal supernova remnants (magenta, SNR). The crosses indicate calculated colours for sources in our sample for which we have no spectra.

\subsection{The rest of the sources}

For the rest of the sources, we do not obtain any strong evidence for AGN activity. $L_{X}$ values are not high enough to independently suggest AGN activity, but this is expected as we are looking for hidden AGN. The lack of adequate spectra makes it impossible to search for $\mathrm{Fe} \mathrm{K} \alpha$ emission. We only get a hint as to the nature of these sources by searching $\Gamma-N_{\mathrm{H}}^{\text {int }}$ space for pairs of values that will reproduce the X-ray colours, H1 and $\mathrm{H} 2$, calculated from the image. The location of the sources in the H1-H2 plane (Fig. 7) provides some insight as to their possible XRB nature. Otherwise, since neither $\Gamma$ nor $N_{\mathrm{H}}^{\text {int }}$ can be established independently, this procedure cannot be used to claim detection of a hidden AGN. In any case, this technique suggests that one of these sources is consistent with an AGN with high intrinsic absorption. All sources are consistent either with lowor high-mass XRBs. X-ray spectra would be needed to further explore these possibilities. Such spectra would further allow to differentiate between ULXs and AGN. However, a defining characteristic of ULXs is their off-nuclear nature, and all of these sources appear to be nuclear.

\subsection{The starburst-AGN connection}

There appears to be an intimate link between starbursts and AGN which is not yet well understood. One the one hand, high $L_{X}$ in composite galaxies suggests an AGN, although based on their optical spectra these are classified, at best, as being on the border between AGN and star-forming galaxies. On the other hand, we detect LLAGN in bona fidæ star-forming galaxies. All these objects have one common trait: they include a powerful starburst, indicative of a large gas supply to feed a central engine. In our sample, the most significant detection of $6.4 \mathrm{keV}$ emission comes from the source with highest $\mathrm{H} \alpha$-calculated SFR. A disturbed morphology, including bars and recent or on-going interactions are further clues to an efficient feeding mechanism.

This does not imply that all such cases will at present accrete onto a super-massive black hole. In the sample of 
Barger et al. (2001) luminous hard X-ray sources are common in bulge-dominated optically luminous galaxies, with about $10 \%$ of the population showing activity at any given time. These authors suggest that this fraction may be interpreted in terms of a duty cycle of galaxies, which are active at any given time.

Alternatively, an evolutionary scheme such as the one proposed by Mouri \& Taniguchi (2004) will conceivably limit the number of sources that actually have an active component at any given time, irrespective of power. We may then simply be detecting galaxies which, according to the terminology of these authors, are in a "starburst dominant" or "starburst-dominant Seyfert" phase, whereas galaxies lacking an AGN signature may be in an "inactive" phase. Put differently, there is a continuum of galactic "activity" levels, and the level of the galaxies with a hidden AGN detected in our work is lower than the galaxies of Cid Fernandes et al. (2001). In the case of NGC 253, which like our sources, also has low $L_{\mathrm{X}}$ and line emission suggesting a buried AGN, Weaver et al. (2002) suggest that one may be detecting the ending or beginning of AGN activity.

Although it might be instructive to do so, we have not attempted to use infra-red diagnostics of AGN/star-forming activity, such as $16 \mu / 24 \mu$ or $70 \mu / 24 \mu$ flux ratios. However, given the low level of AGN activity in these objects, it would be surprising if such tools were to provide any further insight.

\section{Summary}

We used Chandra data to look for X-ray signatures of AGN in an optically selected sample of star-forming galaxies from HFS. We identified and isolated 10 individual X-ray counterparts to optical nuclear sources. We produced X-ray spectra for four of these, and used X-ray colours to explore $\Gamma-N_{\mathrm{H}}^{\text {int }}$ space for the rest. We estimated SFRs, using $\mathrm{H} \alpha$ luminosities. Our main conclusions are:

1. All sources have low $L_{\mathrm{X}}$, not indicative of powerful AGN.

2. For the strong starbursts NGC 2782 and NGC 3310, we detect a $6.4 \mathrm{keV}$ Fe $\mathrm{K} \alpha$ line with high significance. However, these AGN have significantly sub-Eddington luminosities, whilst the level of contamination of the total galactic X-ray flux is small. NGC 2782 has the highest SFR, and NGC 3310 the fourth highest in our sample.

3. In spite of the lack of $\mathrm{Fe} \mathrm{K} \alpha$ line emission, the powerful starburst NGC 4303 is a good candidate for harbouring a hidden AGN. For sources with no spectra, X-ray colours are consistent with LMXBs (three sources), HMXBs (three sources), or a hidden AGN (one source).

The detection of underlying AGN activity in apparently "pure" star-forming galaxies is a further indication that the two components often co-exist and that there is no clear cut-off below which any form of AGN activity can be ruled out altogether.

Acknowledgements. This work was funded in part by the Hellenic General Secretariat for Research and Technology within the framework of the HellasUSA collaboration programme Study of Galaxies with the Chandra X-ray Satellite. This research has made use of data obtained from the High Energy Astrophysics Science Archive Research Center (HEASARC), provided by NASA's Goddard Space Flight Center. This research has made use of the NASA/IPAC Extragalactic Database (NED) which is operated by the Jet Propulsion Laboratory, California Institute of Technology, under contract with the National Aeronautics and Space Administration.

\section{References}

Arp, H. 1966, ApJS, 14, 1

Balick, B., \& Heckman, T. 1981, A\&A, 96, 271

Ballo, L., Braito, V., Della Ceca, R., et al. 2004, ApJ, 600, 634

Balzano, V. A. 1983, ApJ, 268, 602

Barger, A. J., Cowie, L. L., Mushotzky, R. F., \& Richards, E. A. 2001, AJ, 121, 662

Cid Fernandes, R., Heckman, T., Schmitt, H., Delgado, R. M. G., \& Storchi-Bergmann, T. 2001, ApJ, 558, 81

Colina, L., Garcia Vargas, M. L., Mas-Hesse, J. M., Alberdi, A., \& Krabbe, A. 1997, ApJ, 484, L41

Colina, L., \& Wada, K. 2000, ApJ, 529, 845

Cotton, W. D., Condon, J. J., \& Arbizzani, E. 1999, ApJS, 125, 409

de Vaucouleurs, G., de Vaucouleurs, A., Corwin, H. G., et al. 1991, Third Reference Catalogue of Bright Galaxies, Volume 1-3, XII, 2069, 7 figs. (Berlin, Heidelberg, New York: Springer-Verlag)

Devereux, N. A. 1989, ApJ, 346, 126

Dickey, J. M., \& Lockman, F. J. 1990, ARA\&A, 28, 215

Fabbiano, G., Zezas, A., \& Murray, S. S. 2001, ApJ, 554, 1035

Fabian, A. C., Barcons, X., Almaini, O., \& Iwasawa, K. 1998, MNRAS, 297, L11

Ferrarese, L., \& Ford, H. 2005, Space Sci. Rev., 116, 523

Gebhardt, K., Bender, R., Bower, G., et al. 2000, ApJ, 539, L13

Georgantopoulos, I., \& Georgakakis, A. 2005, MNRAS, 358, 131

González Delgado, R. M., Heckman, T., \& Leitherer, C. 2001, ApJ, 546, 845

Ho, L. C., Filippenko, A. V., \& Sargent, W. L. W. 1997, ApJS, 112, 315

Hornschemeier, A. E., Brandt, W. N., Garmire, G. P., et al. 2001, ApJ, 554, 742

Jenkins, L. P., Roberts, T. P., Ward, M. J., \& Zezas, A. 2004, MNRAS, 352, 1335

Jiménez-Bailón, E., Santos-Lleó, M., Mas-Hesse, J. M., et al. 2003, ApJ, 593, 127

Jogee, S., Kenney, J. D. P., \& Smith, B. J. 1999, ApJ, 526, 665

Kennicutt, R. C. 1998, ARA\&A, 36, 189

Knapen, J. H., Stedman, S., Bramich, D. M., Folkes, S. L., \& Bradley, T. R. 2004, A\&A, 426, 1135

Kregel, M., \& Sancisi, R. 2001, A\&A, 376, 59

Laine, S., Shlosman, I., Knapen, J. H., \& Peletier, R. F. 2002, ApJ, 567, 97

Levenson, N. A., Krolik, J. H., Życki, P. T., et al. 2002, ApJ, 573, L81

Lynden-Bell, D. 1969, Nature, 223, 690

Magorrian, J., Tremaine, S., Richstone, D., et al. 1998, AJ, 115, 2285

McElroy, D. B. 1995, ApJS, 100, 105

Moran, E. C., Helfand, D. J., Becker, R. H., \& White, R. L. 1996, ApJ, 461, 127

Mouri, H., \& Taniguchi, Y. 2004, ApJ, 605, 144

Mukai, K. 1993, Legacy, 3, 21

Nicastro, F., Martocchia, A., \& Matt, G. 2003, ApJ, 589, L13

Panessa, F., Wolter, A., Pellegrini, S., et al. 2005, ApJ, 631, 707

Pastoriza, M. G., Bonatto, C. J., Storchi-Bergmann, T., \& Bica, E. 1993, Rev. Mex. Astron. Astrofis., 26, 124

Pellegrini, S. 2005, ApJ, 624, 155

Pérez-Ramírez, D., Knapen, J. H., Peletier, R. F., et al. 2000, MNRAS, 317, 234 Prestwich, A. H., Irwin, J. A., Kilgard, R. E., et al. 2003, ApJ, 595, 719

Protassov, R., van Dyk, D. A., Connors, A., Kashyap, V. L., \& Siemiginowska, A. 2002, ApJ, 571,545

Rees, M. J. 1986, in Structure and Evolution of Active Galactic Nuclei, ed. G. Giuricin, M. Mezzetti, M. Ramella, \& F. Mardirossian, ASSL, 121, 447

Reynolds, C. S., \& Nowak, M. A. 2003, Phys. Rept., 377, 389

Salpeter, E. E. 1964, ApJ, 140, 796

Schulz, H., Komossa, S., Berghofer, T. W., \& Boer, B. 1998, A\&A, 330, 823

Smith, B. J. 1991, ApJ, 378, 39

Smith, B. J. 1994, AJ, 107, 1695

Smith, B. J., Struck, C., Kenney, J. D. P., \& Jogee, S. 1999, AJ, 117, 1237

Smith, D. A., Neff, S. G., Bothun, G. D., et al. 1996, ApJ, 473, L21

Strickland, D. K. 2005, in Extra-Planar Gas, ed. R. Braun, ASP Conf. Ser., 331, 345

Terashima, Y., Ho, L. C., Ptak, A. F., et al. 2000, ApJ, 535, L79

Terashima, Y., Iyomoto, N., Ho, L. C., \& Ptak, A. F. 2002, ApJS, 139, 1

Tschöke, D., Hensler, G., \& Junkes, N. 2000, A\&A, 360, 447

Véron-Cetty, M.-P., \& Véron, P. 2003, A\&A, 412, 399

Wang, Q. D., Gotthelf, E. V., \& Lang, C. C. 2002, Nature, 415, 148

Weaver, K. A., Heckman, T. M., Strickland, D. K., \& Dahlem, M. 2002, ApJ, 576, L19

Wehner, E. H., \& Gallagher, J. S. 2005, ApJ, 618, L21

Zezas, A. L., Georgantopoulos, I., \& Ward, M. J. 1998, MNRAS, 301, 915 